\title{
Sciendo
}

\author{
BULGARIAN ACADEMY OF SCIENCES
}

CYBERNETICS AND INFORMATION TECHNOLOGIES • Volume 21, No 4

Sofia $2021 \quad$ Print ISSN: 1311-9702; Online ISSN: 1314-4081

DOI: $10.2478 /$ cait-2021-0047

\section{Modal Type of Weak Intuitionistic Fuzzy Implications Generated by the Operation $\triangle$}

\author{
Piotr Dworniczak ${ }^{1}$, Lilija Atanassova ${ }^{2}$, Nora Angelova ${ }^{3}$ \\ ${ }^{1}$ ORCID: 0000-0001-8904-9115, The Great Poland University of Social and Economics, ul. Surzyńskich \\ 2, 63-000 Środa Wlkp., Poland \\ ${ }^{2}$ ORCID: 0000-0002-5173-5256, Institute of Information and Communication Technologies, Bulgarian \\ Academy of Sciences, Acad. G. Bonchev Str., Bl. 2, 1113 Sofia, Bulgaria \\ ${ }^{3}$ ORCID: 0000-0003-2697-9766, Faculty of Mathematics and Informatics, Sofia University "St. Kliment \\ Ohridski", 5 James Bourchier Blvd., 1164 Sofia, Bulgaria \\ E-mails:p.dworniczak@wwsse.pl l.c.atanassova@gmail.com noraa@fmi.uni-sofia.bg
}

\begin{abstract}
In 2020 L. Atanassova has been introduced the new operation $\triangle$ over intuitionistic fuzzy sets and over intuitionistic fuzzy pairs. Some of its properties have been studied in 2021 from L. Atanassova and P. Dworniczak. In 2021 L. Atanassova and $P$. Dworniczak generated an intuitionistic fuzzy implication by the operation $\triangle$, and it has been introduced and some of its basic properties have been described. Here, eight modal type of intuitionistic fuzzy implications are generated by the first one and some of their properties are discussed.
\end{abstract}

Keywords: Intuitionistic fuzzy implication, intuitionistic fuzzy operation, intuitionistic fuzzy operator.

2020 Mathematics Subject Classification: 03E72, 47S40.

\section{Introduction}

Many operations have been introduced over Intuitionistic Fuzzy Sets (IFSs, see $[1,2]$ ) and over Intuitionistic Fuzzy Pairs (IFPs, see [4]) and their properties have been studied. In [5], the new operation $\triangle$ has been introduced over two IFSs or over two IFPs. Some of its properties have been described in [6].

In the present research, eight intuitionistic fuzzy implications, which are generated by the above-mentioned operation will be defined and some of their properties will be discussed.

\section{Preliminaries}

In [4] K. Atanassov, E. Szmidt and J. Kacprzyk called the object $\langle a, b\rangle$ an Intuitionistic Fuzzy Pair (IFP), where $a, b, a+b \in[0,1]$. 
Let everywhere below for IFPs $x$ and $y$ :

$$
\begin{aligned}
& x=\langle a, b\rangle, \\
& y=\langle c, d\rangle,
\end{aligned}
$$

where $a, b, c, d, a+b, c+d \in[0,1]$. For the IFPs, in [4] different operations and relations are defined. For our aims, we will give the definitions of only three relations and one operation:

$$
\begin{gathered}
x \leq y \text { if and only if } a \leq c \text { and } b \geq d, \\
x \geq y \text { if and only if } y \leq x, \\
x=y \text { if and only if } x \leq y \text { and } y \leq x, \\
\neg x=\langle b, a\rangle .
\end{gathered}
$$

\section{Main results}

The implication from [7] is defined by

$$
x \rightarrow y=\left\langle\frac{b+c}{a+b+c+d}, \frac{a+d}{a+b+c+d}\right\rangle
$$

for $a+b+c+d>0$ and

$$
\langle 0,0\rangle \rightarrow\langle 0,0\rangle=\left\langle\frac{1}{2}, \frac{1}{2}\right\rangle .
$$

The (standard) intuitionistic fuzzy modal operators over IFPs (see [3]) are

$$
\begin{aligned}
& \square x=\langle a, 1-a\rangle, \\
& \diamond x=\langle 1-b, b\rangle .
\end{aligned}
$$

The intuitionistic fuzzy modal operators of second type over IFPs (see [3]) are

$$
\begin{aligned}
& \boxplus x=\left\langle\frac{a}{2}, \frac{b+1}{2}\right\rangle, \\
& \nabla x=\left\langle\frac{a+1}{2}, \frac{b}{2}\right\rangle .
\end{aligned}
$$

Now, using the logical scheme (see, e.g., $[3,8]$ )

(1)

$$
x \rightarrow * y=M_{1} x \rightarrow M_{2} y,
$$

where $M_{1}, M_{2} \in\{\square, \diamond\}$, we define

$$
\begin{aligned}
& x \rightarrow_{1} y=\square x \rightarrow \diamond y=\langle a, 1-a\rangle \rightarrow\langle 1-d, d\rangle=\left\langle\frac{2-a-d}{2}, \frac{a+d}{2}\right\rangle= \\
& =\left\langle 1-\frac{a+d}{2}, \frac{a+d}{2}\right\rangle \text {, } \\
& x \rightarrow_{2} y=\square x \rightarrow \square y=\langle a, 1-a\rangle \rightarrow\langle c, 1-c\rangle=\left\langle\frac{1-a+c}{2}, \frac{1+a-c}{2}\right\rangle, \\
& x \rightarrow_{3} y=\diamond x \rightarrow \diamond y=\langle 1-b, b\rangle \rightarrow\langle 1-d, d\rangle=\left\langle\frac{1+b-d}{2}, \frac{1-b+d}{2}\right\rangle,
\end{aligned}
$$




$$
\begin{aligned}
x \rightarrow_{4} y=\diamond x \rightarrow \square y= & \langle 1-b, b\rangle \rightarrow\langle c, 1-c\rangle=\left\langle\frac{b+c}{2}, \frac{2-b-c}{2}\right\rangle= \\
& =\left\langle\frac{b+c}{2}, 1-\frac{b+c}{2}\right\rangle .
\end{aligned}
$$

For these four new implications, we can prove the following assertions.

Theorem 1. For every two IFPs $x$ and $y$

$$
x \rightarrow_{1} y \geq\left\{\begin{array}{l}
x \rightarrow_{2} y \\
x \rightarrow_{3} y
\end{array}\right\} \geq x \rightarrow_{4} y .
$$

Proof:

Let the two IFPs $x$ and $y$ be given. Then from the inequalities

$$
\begin{gathered}
1-\frac{a+d}{2} \geq \frac{1-a+c}{2}, \\
\frac{a+d}{2} \leq \frac{1+a-c}{2}, \\
1-\frac{a+d}{2} \geq \frac{1+b-d}{2}, \\
\frac{a+d}{2} \leq \frac{1-b+d}{2}, \\
\frac{1-a+c}{2} \geq \frac{b+c}{2}, \\
\frac{1+a-c}{2} \leq 1-\frac{b+c}{2}, \\
\frac{1+b-d}{2} \geq \frac{b+c}{2}, \\
\frac{1-b+d}{2} \leq 1-\frac{b+c}{2},
\end{gathered}
$$

it follows the validity of Theorem 1 .

Theorem 2. For every two IFPs $x$ and $y$

Proof:

$$
\begin{aligned}
& \neg x \rightarrow_{1} \neg y=\neg\left(x \rightarrow_{4} y\right), \\
& \neg x \rightarrow_{2} \neg y=\neg\left(x \rightarrow_{3} y\right), \\
& \neg x \rightarrow_{3} \neg y=\neg\left(x \rightarrow_{2} y\right), \\
& \neg x \rightarrow_{4} \neg y=\neg\left(x \rightarrow_{1} y\right) .
\end{aligned}
$$

We check the first equality and the validity of the rest three equalities is checked in the same manner.

$$
\begin{aligned}
& \neg x \rightarrow_{1} \neg y=\neg\langle a, b\rangle \rightarrow_{1} \neg\langle c, d\rangle=\langle b, a\rangle \rightarrow_{1}\langle d, c\rangle= \\
& =\left\langle 1-\frac{b+c}{2}, \frac{b+c}{2}\right\rangle=\neg\left\langle\frac{b+c}{2}, 1-\frac{b+c}{2}\right\rangle=\neg\left(x \rightarrow_{4} y\right) .
\end{aligned}
$$


Now, for the scheme (1) we use $M_{1}, M_{2} \in\{\boxplus, \otimes\}$ and receive the following four new intuitionistic fuzzy implications:

$$
\begin{gathered}
x \rightarrow_{5} y=\boxplus x \rightarrow \nabla y=\left\langle\frac{a}{2}, \frac{b+1}{2}\right\rangle \rightarrow\left\langle\frac{c+1}{2}, \frac{d}{2}\right\rangle= \\
=\left\langle\frac{\frac{b+1}{2}+\frac{c+1}{2}}{\frac{a}{2}+\frac{b+1}{2}+\frac{c+1}{2}+\frac{d}{2}}, \frac{\frac{a}{2}+\frac{d}{2}}{\frac{a}{2}+\frac{b+1}{2}+\frac{c+1}{2}+\frac{d}{2}}\right\rangle=\left\langle\frac{b+c+2}{a+b+c+d+2}, \frac{a+d}{a+b+c+d+2}\right\rangle, \\
x \rightarrow_{6} y=\boxplus x \rightarrow \boxplus y=\left\langle\frac{a}{2}, \frac{b+1}{2}\right\rangle \rightarrow\left\langle\frac{c}{2}, \frac{d+1}{2}\right\rangle= \\
=\left\langle\frac{b+c+1}{a+b+c+d+2}, \frac{a+d+1}{a+b+c+d+2}\right\rangle, \\
x \rightarrow_{7} y=\otimes x \rightarrow \nabla y=\left\langle\frac{a+1}{2}, \frac{b}{2}\right\rangle \rightarrow\left\langle\frac{c+1}{2}, \frac{d}{2}\right\rangle= \\
=\left\langle\frac{b+c+1}{a+b+c+d+2}, \frac{a+d+1}{a+b+c+d+2}\right\rangle, \\
x \rightarrow_{8} y=\otimes x \rightarrow \boxplus y=\left\langle\frac{a+1}{2}, \frac{b}{2}\right\rangle \rightarrow\left\langle\frac{c}{2}, \frac{d+1}{2}\right\rangle= \\
=\left\langle\frac{b+c}{a+b+c+d+2}, \frac{a+d+2}{a+b+c+d+2}\right\rangle .
\end{gathered}
$$

Now we formulate Theorems 3 and 4 that are proved in the same manner as Theorems 1 and 2. They have the following forms.

Theorem 3. For every two IFPs $x$ and $y$

$$
x \rightarrow_{5} y \geq x \rightarrow_{6} y=x \rightarrow_{7} y \geq x \rightarrow_{8} y .
$$

Theorem 4. For every two IFPs $x$ and $y$

$$
\begin{aligned}
& \neg x \rightarrow_{5} \neg y=\neg\left(x \rightarrow_{8} y\right), \\
& \neg x \rightarrow_{8} \neg y=\neg\left(x \rightarrow_{5} y\right) .
\end{aligned}
$$

We check directly the validity of the following equalities:

$$
\begin{gathered}
\langle 0,1\rangle \rightarrow_{i}\langle 0,1\rangle= \begin{cases}\left\langle\frac{1}{2}, \frac{1}{2}\right\rangle & \text { for } i=1,2,3,4,6,7, \\
\left\langle\frac{3}{4}, \frac{1}{4}\right\rangle & \text { for } i=5, \\
\left\langle\frac{1}{4}, \frac{3}{4}\right\rangle & \text { for } i=8,\end{cases} \\
\langle 0,1\rangle \rightarrow_{i}\langle 0,0\rangle=\left\{\begin{array}{lll}
\langle 1,0\rangle & \text { for } i=1,3,5, \\
\left\langle\frac{1}{2}, \frac{1}{2}\right\rangle & \text { for } i=2,4, \\
\left\langle\frac{2}{3}, \frac{1}{3}\right\rangle & \text { for } i=6,7, \\
\left\langle\frac{1}{3}, \frac{2}{3}\right\rangle & \text { for } i=8,
\end{array}\right.
\end{gathered}
$$




$$
\begin{aligned}
& \langle 0,1\rangle \rightarrow_{i}\langle 1,0\rangle= \begin{cases}\langle 1,0\rangle & \text { for } i=1,2,3,4,5, \\
\left\langle\frac{3}{4}, \frac{1}{4}\right\rangle & \text { for } i=6,7, \\
\left\langle\frac{1}{2}, \frac{1}{2}\right\rangle & \text { for } i=8,\end{cases} \\
& \langle 0,0\rangle \rightarrow_{i}\langle 0,1\rangle=\left\{\begin{array}{lll}
\left\langle\frac{1}{2}, \frac{1}{2}\right\rangle & \text { for } i=1,2, \\
\langle 0,1\rangle & \text { for } i=3,4,8, \\
\left\langle\frac{2}{3}, \frac{1}{3}\right\rangle & \text { for } i=5, \\
\left\langle\frac{1}{3}, \frac{2}{3}\right\rangle & \text { for } i=6,7,
\end{array}\right. \\
& \langle 0,0\rangle \rightarrow_{i}\langle 0,0\rangle=\left\{\begin{array}{lll}
\langle 1,0\rangle & \text { for } & i=1,5, \\
\left\langle\frac{1}{2}, \frac{1}{2}\right\rangle & \text { for } & i=2,3,6,7, \\
\langle 0,1\rangle & \text { for } & i=4,8,
\end{array}\right. \\
& \langle 0,0\rangle \rightarrow_{i}\langle 1,0\rangle=\left\{\begin{array}{lll}
\langle 1,0\rangle & \text { for } i=1,2,5, \\
\left\langle\frac{1}{2}, \frac{1}{2}\right\rangle & \text { for } i=3,4, \\
\left\langle\frac{2}{3}, \frac{1}{3}\right\rangle & \text { for } i=6,7, \\
\left\langle\frac{1}{3}, \frac{2}{3}\right\rangle & \text { for } i=8,
\end{array}\right. \\
& \langle 1,0\rangle \rightarrow_{i}\langle 0,1\rangle= \begin{cases}\langle 0,1\rangle & \text { for } i=1,2,3,4,8, \\
\left\langle\frac{1}{2}, \frac{1}{2}\right\rangle & \text { for } i=5, \\
\left\langle\frac{1}{4}, \frac{3}{4}\right\rangle & \text { for } \quad i=6,7,\end{cases} \\
& \langle 1,0\rangle \rightarrow_{i}\langle 0,0\rangle=\left\{\begin{array}{lll}
\left\langle\frac{1}{2}, \frac{1}{2}\right\rangle & \text { for } \quad i=1,3, \\
\langle 0,1\rangle & \text { for } i=2,4,8, \\
\left\langle\frac{2}{3}, \frac{1}{3}\right\rangle & \text { for } \quad i=5, \\
\left\langle\frac{1}{3}, \frac{2}{3}\right\rangle & \text { for } \quad i=6,7,
\end{array}\right. \\
& \langle 1,0\rangle \rightarrow_{i}\langle 1,0\rangle= \begin{cases}\left\langle\frac{1}{2}, \frac{1}{2}\right\rangle & \text { for } i=1,2,3,4,6,7, \\
\left\langle\frac{3}{4}, \frac{1}{4}\right\rangle & \text { for } i=5, \\
\left\langle\frac{1}{4}, \frac{3}{4}\right\rangle & \text { for } i=8 .\end{cases}
\end{aligned}
$$

Finally we formulate the following assertion that is proved as the above ones.

Theorem 5. For every two IFPs $x$ and $y$

$$
x \rightarrow_{5} y \geq x \rightarrow_{1} y \geq x \rightarrow_{4} y \geq x \rightarrow_{8} y .
$$

For any $x$ let us define the degree of definiteness $h(x)$ (see [1, p. 127], and [2, p. 134]) called the accuracy degree also, as follows:

$$
h(x)=a+b .
$$


Then, we can formulate another assertion.

Theorem 6. For every two IFPs $x$ and $y$

$$
\begin{aligned}
& h(x) \geq h(y) \text { if and only if } x \rightarrow_{3} y \geq x \rightarrow_{2} y, \\
& h(x) \leq h(y) \text { if and only if } x \rightarrow_{3} y \leq x \rightarrow_{2} y .
\end{aligned}
$$

The proofs of this and of the assertions in the next section are in the same manner as of Theorem 1.

\section{Negations generated by the new implications}

Following [3] and basing, e.g., on [10], we mention that

$$
\neg x=x \rightarrow\langle 0,1\rangle \text {. }
$$

Therefore, the discussed in the paper implications generate the following negations:

$$
\begin{gathered}
\neg_{0} x=x \rightarrow\langle 0,1\rangle=\left\langle\frac{b}{a+b+1}, \frac{a+1}{a+b+1}\right\rangle, \\
\neg_{1} x=x \rightarrow_{1}\langle 0,1\rangle=\left\langle\frac{1-a}{2}, \frac{a+1}{2}\right\rangle, \\
\neg_{2} x=x \rightarrow_{2}\langle 0,1\rangle=\left\langle\frac{1-a}{2}, \frac{a+1}{2}\right\rangle, \\
\neg_{3} x=x \rightarrow_{3}\langle 0,1\rangle=\left\langle\frac{b}{2}, 1-\frac{b}{2}\right\rangle, \\
\neg_{4} x=x \rightarrow_{4}\langle 0,1\rangle=\left\langle\frac{b}{2}, 1-\frac{b}{2}\right\rangle, \\
\neg_{5} x=x \rightarrow_{5}\langle 0,1\rangle=\left\langle\frac{b+2}{a+b+3}, \frac{a+1}{a+b+3}\right\rangle, \\
\neg_{6} x=x \rightarrow_{6}\langle 0,1\rangle=\left\langle\frac{b+1}{a+b+3}, \frac{a+2}{a+b+3}\right\rangle, \\
\neg_{7} x=x \rightarrow_{7}\langle 0,1\rangle=\left\langle\frac{b+1}{a+b+3}, \frac{a+2}{a+b+3}\right\rangle, \\
\neg_{8} x=x \rightarrow_{8}\langle 0,1\rangle=\left\langle\frac{b}{a+b+3}, \frac{a+3}{a+b+3}\right\rangle .
\end{gathered}
$$

Theorem 7. For each IFP $x$

$$
\begin{gathered}
\neg_{5} x \geq \neg_{6} x=\neg_{7} x \geq \neg_{8} x, \\
\neg_{5} x \geq \neg_{1} x=\neg_{2} x \geq \neg_{0} x \geq \neg_{3} x=\neg_{4} x \geq \neg_{8} x .
\end{gathered}
$$

5. The new implications and the axioms of G. Klir and B. Yuan

G. K1ir and B. Yuan [9] have defined nine axioms for fuzzy implication. K. A t a n a s o v [3] pre-formulated them for the case of intuitionistic fuzziness. 
Let

$$
\begin{aligned}
& O^{*}=\langle 0,1\rangle, \\
& E^{*}=\langle 1,0\rangle .
\end{aligned}
$$

The IFP is an Intuitionistic Fuzzy Tautology (IFT) if and only if $a \geq b$.

G. Klir and B. Yuan's axioms are:

Axiom A1 $(\forall x, y)($ if $x \leq y$, then $(\forall z)(x \rightarrow z \geq y \rightarrow z))$,

Axiom A2 $(\forall x, y)($ if $x \leq y$, then $(\forall z)(z \rightarrow x \leq z \rightarrow y))$,

Axiom A3 $(\forall y)\left(O^{*} \rightarrow y=E^{*}\right)$,

Axiom A4 $(\forall y)\left(E^{*} \rightarrow y=y\right)$,

Axiom A5 $(\forall x)\left(x \rightarrow x=E^{*}\right)$,

Axiom A6 $(\forall x, y, z)(x \rightarrow(y \rightarrow z)=y \rightarrow(x \rightarrow z))$,

Axiom A7 $(\forall x, y)\left(x \rightarrow y=E^{*}\right.$ if and only if $\left.x \leq y\right)$,

Axiom A8 $(\forall x, y)(x \rightarrow y=\neg y \rightarrow \neg x)$,

Axiom A9 $\rightarrow$ is a continuous function.

In [3], having in mind the specific forms of the intuitionistic fuzzy implications, K. Atanassov modified five of these axioms, as follows:

Axiom A3* $\quad(\forall y)\left(O^{*} \rightarrow y\right.$ is an IFT $)$,

Axiom A4* $\quad(\forall y)\left(E^{*} \rightarrow y \leq y\right)$,

Axiom A5* $\quad(\forall x)(x \rightarrow x$ is an IFT $)$,

Axiom A7* $\quad(\forall x, y)\left(\right.$ if $x \leq y$, then $\left.x \rightarrow y=E^{*}\right)$,

Axiom A8* $\quad(\forall x, y)(x \rightarrow y=\neg(\neg(\neg y \rightarrow \neg x)))$.

Theorem 8. For every three IFPs $x, y$ and $z$ :

(a) All implications without $\rightarrow_{8}$ satisfy Axioms A1, A2, A3* as IFTs,

(b) Implications $\rightarrow_{2}, \rightarrow_{4}$ satisfy Axiom A4* as a tautology,

(c) Implications $\rightarrow_{1}, \rightarrow_{2}, \rightarrow_{3}, \rightarrow_{5}, \rightarrow_{6}, \rightarrow_{7}$ satisfy Axiom A5* as a tautology,

(d) Only implication $\rightarrow_{5}$ satisfies Axiom A7* as a tautology,

(e) All implications satisfy Axioms A9.

Proof:

(a) Let $x, y$ and $z$ be IFPs and let $x \leq y$, i.e., $a \leq c$ and $b \geq d$. Then for Axiom A1 we obtain:

$$
x \rightarrow_{1} z=\left\langle 1-\frac{a+f}{2}, \frac{a+f}{2}\right\rangle \geq\left\langle 1-\frac{c+f}{2}, \frac{c+f}{2}\right\rangle=y \rightarrow_{1} z .
$$

On the other hand, for Axiom A4*

and in spite of

$$
E^{*} \rightarrow_{1} y=\left\langle 1-\frac{1+d}{2}, \frac{1+d}{2}\right\rangle,
$$

$$
\frac{1+d}{2} \geq d
$$

for each $d \in[0,1]$, obviously,

$$
1-\frac{1+d}{2} \leq c
$$


is not always valid, because for $c=0, d<1$ this inequality is not true. Therefore, implication $\rightarrow_{1}$ does not satisfy Axiom A4*.

All other assertions are checked similarly.

\section{Conclusion}

In the present paper, new eight intuitionistic fuzzy implications are introduced and some of their properties are studied. In a next research, we will check which axioms of Klir and Yuan's , Kolmogorov's, Lukasiewicz and Tarski's axiom for implications and which intuitionistic logic axioms are valid for the first and the new eight implications.

\section{References}

1. A t a n a s s o v, K. Intuitionistic Fuzzy Sets. Springer, Heidelberg, 1999.

2. A t a n a s s o v, K. On Intuitionistic Fuzzy Sets Theory. Springer, Berlin, 2012.

3. A t a n a s o v, K. Intuitionistic Fuzzy Logics. Springer, Cham, 2017.

4. A t a n a s s o v, K. T., E. S z midt, J. K a c pr z y k. On Intuitionistic Fuzzy Pairs. - Notes on Intuitionistic Fuzzy Sets, Vol. 19, 2013, No 3, pp. 1-13.

5. A t a n a s s o v a, L. A New Operator over Intuitionistic Fuzzy Sets. - Notes on Intuitionistic Fuzzy Sets, Vol. 26, 2020, No 1, pp. 23-28.

6. Atanas sova, L., P. D w orniczak. On the Operation $\triangle$ over Intuitionistic Fuzzy Sets. Mathematics, Vol. 9, 2021, No 13, p. 1518

7. A t a $\mathrm{n}$ a s s o v a, L., P. D w o r $\mathrm{n}$ i $\mathrm{z}$ a k. On the Weak Intuitionistic Fuzzy Implication Based on $\triangle$ Operation. - Notes on Intuitionistic Fuzzy Sets, Vol. 27, 2021, No 2, pp. 11-19.

8. F e y s, R. Modal Logics. Gauthier-Villars, Paris, 1965.

9. K 1 i r, G., B. Y u a n. Fuzzy Sets and Fuzzy Logic. Prentice Hall, New Jersey, 1995.

10. M e n d e 1 s o n, E. Introduction to Mathematical Logic. 6th Ed. CRC Press, Boca Raton, FL, USA, 2015.

Received: 31.07.2021; Second Version: 12.09.2021; Accepted: 04.10.2021 